\title{
ON THE STABILITY OF THE SPHERICAL SHAPE OF A VAPOR CAVITY IN A LIQUID*
}

\author{
BY \\ M. S. PLESSET AND T. P. MITCHELL \\ California Institute of T'echnology
}

1. Introduction. It has been shown by G. I. Taylor [1] that a plane interface between two fluids of different densities in accelerated motion is stable or unstable according as the acceleration is directed from the heavier to the lighter fluid, or conversely. This stability analysis is limited to small amplitude perturbations of the plane interface; and it is found that a small distortion of the interface begins to grow exponentially with time in the unstable situation and to decrease exponentially in the stable situation. While experimental observations agree well with the theory in the small amplitude limit for which the theory is valid, it is known that there are significant deviations in the rate of growth of distortions in the unstable case when their amplitudes become appreciable [2].

It is of interest to consider the analogous stability problem for the case of a spherical interface between two immiscible fluids of different densities in accelerated motion. For perturbations in the spherical interface of small amplitude, it may be shown [3] that the stability criterion deduced by Taylor for the plane case is subject to important modifications.

The stability problem in the spherical case may be formulated as follows. A fluid of density $\rho_{1}$ is contained within a sphere of radius $R$; a fluid of density $\rho_{2}$ occupies the region exterior to this sphere. The fluids are supposed to be immiscible, incompressible and nonviscous. The equation of motion for the interface radius as a function of time, $R(t)$, is readily determined under the assumption that the initial and boundary conditions are spherically symmetric. If the interface is distorted from the surface of a sphere of radius $R$ to a surface with radius vector of magnitude $r_{\mathbf{0}}$, then one may write

$$
r_{s}=R+\sum a_{n} Y_{n}
$$

where $Y_{n}$ is a spherical harmonic of degree $n$ and the $a_{n}$ 's are functions of the time to be determined. The stability of the spherical interface may be established by considering whether interface distortions of small amplitude grow or diminish. More precisely, it is assumed that

$$
\left|a_{n}(t)\right| \ll R(t),
$$

and that terms of order higher than the first in $a_{n}$ and $d a_{n} / d t$ are negligible. In such a linearized perturbation theory, the $a_{n}$ 's are independent of each other, and it may be shown [3] that they satisfy the following differential equation

$$
\frac{d^{2} a_{n}}{d t^{2}}+\frac{3}{R} \frac{d R}{d t} \frac{d a_{n}}{d t}-A a_{n}=0
$$

with

$$
A=\frac{\left[n(n-1) \rho_{2}-(n+1)(n+2) \rho_{1}\right] d^{2} R / d t^{2}-(n-1) n(n+1)(n+2) \sigma / R^{2}}{\left[n \rho_{2}+(n+1) \rho_{1}\right] R},
$$

where $\sigma$ is the surface tension constant.

${ }^{*}$ Received October 11, 1954. This study was supported by the U. S. Office of Naval Research. 
The spherical stability problem has application to the behavior of growing or collapsing gas bubbles in a liquid. Penney and Price [4] have carried out a numerical solution of the stability equation (2) for $n=2$ for the case of a pulsating gas bubble in water with an internal pressure, $p_{i}$, in the bubble given by

$$
p_{i} R^{3 \gamma}=\text { const. }
$$

and with a constant pressure, $p_{0}$, in the liquid at a distance from the bubble. In their computations surface tension is neglected. The numerical solution showed that the distortion amplitude $a_{2}$ is much larger when the bubble is near its minimum radius than elsewhere. The problem to be considered here is a cavity for which the internal pressure, $p_{i}$, is constant, in a liquid at constant external pressure $p_{0}$. These are approximately the conditions in a vapor cavitation bubble in a liquid. An analytic solution for the stability equation may be found under these conditions.

2. Solution of the stability problem. For a vapor cavity in a liquid, the vapor density $\rho_{1}$ may be neglected in comparison with the liquid density $\rho_{2}$. The quantity $A$ of Eq. (3) then becomes

$$
A=\frac{(n-1)}{R} \frac{d^{2} R}{d t^{2}}-(n-1)(n+1)(n+2) \frac{\sigma}{\rho R^{3}},
$$

where $\rho=\rho_{2}$ is the liquid density. The equation of motion of the undisturbed interface $[3]$ is

$$
R \frac{d^{2} R}{d t^{2}}+\frac{3}{2}\left(\frac{d R}{d t}\right)^{2}=\frac{p_{i}-p_{0}-2 \sigma / R}{\rho} .
$$

Equation (5) may also be written as

$$
\frac{d}{d t}\left[R^{3}\left(\frac{d R}{d t}\right)^{2}\right]=2 R^{2} \frac{d R}{d t}\left[\frac{p_{i}-p_{0}-2 \sigma / R}{\rho}\right]
$$

which integrates, when $p_{v}-p_{0}$ is a constant, to give

$$
2 \pi \rho\left[R^{3}\left(\frac{d R}{d t}\right)^{2}-R_{0}^{3}\left(\frac{d R_{0}}{d t}\right)^{2}\right]=\frac{4 \pi}{3}\left(p_{i}-p_{0}\right)\left(R^{3}-R_{0}^{3}\right)-4 \pi \sigma\left(R^{2}-R_{0}^{2}\right),
$$

where $R_{0}$ is the cavity radius and $d R_{0} / d t$ is its radial velocity at $t=t_{0}$. This integral of Eq. (5) is to be recognized as the energy integral of the system.

The general features of the asymptotic behavior of the distortion amplitude $a_{n}$ may be made evident in a straightforward way. For the case of an expanding bubble, Eq. (6) gives

$$
\left(\frac{d R}{d t}\right)^{2} \sim \frac{2 P}{3 \rho}, \quad R \rightarrow \infty
$$

with $P=p_{i}-p_{0}>0$, and it then follows from Eqs. (5) and (2) that

$$
a_{n} \rightarrow \text { const., } \quad R \rightarrow \infty .
$$

For the case of a collapsing bubble, it is convenient to transform Eq. (2) by the substitution

$$
a_{n}=\left(\frac{R_{0}}{R}\right)^{3 / 2} b_{n}
$$


into

with

$$
\frac{d^{2} b_{n}}{d t^{2}}-G(t) b_{n}=0
$$

$$
\begin{aligned}
G(t) & =\frac{3}{2} \frac{d}{d t}\left[\frac{1}{R} \frac{d R}{d t}\right]+\frac{9}{4}\left[\frac{1}{R} \frac{d R}{d t}\right]^{2}+A \\
& =\frac{3}{4} \frac{1}{R^{2}}\left(\frac{d R}{d t}\right)^{2}+\frac{(n+1 / 2)}{R} \frac{d^{2} R}{d t^{2}}-(n-1)(n+1)(n+2) \frac{\sigma}{\rho R^{3}} .
\end{aligned}
$$

From Eq. (6), one has

$$
\left[\frac{d R}{d t}\right]^{2}=\frac{R_{0}^{3}}{R^{3}}\left[\left(\frac{d R_{0}}{d t}\right)^{2}+\frac{2 p}{3 \rho}+\frac{2 \sigma}{\rho R_{0}}\right]+0\left[\frac{1}{R}\right]
$$

where $p=p_{0}-p_{i}>0$ for this case. The radial acceleration, $d^{2} R / d t^{2}$, is determined by Eq. (5); and the function $G(t)$ is found to be

$$
G(t) \sim-\frac{3 n}{2} \frac{R_{0}^{3}}{R^{5}}\left[\left(\frac{d R_{0}}{d t}\right)^{2}+\frac{2 p}{3 \rho}+\frac{2 \sigma}{\rho R_{0}}\right], \quad R \rightarrow 0,
$$

except for smaller terms. It is evident that

$$
G(t) \sim-\frac{n c^{2}}{R^{5}}
$$

where $c$ is a real constant. One may now write a W.K.B. approximation to the solution of Eq. (10) for small $R$ in the form

$$
b_{n} \sim[G(t)]^{-1 / 4} \exp \left\{ \pm \int^{t}\left[G\left(t^{\prime}\right)\right]^{1 / 2} d t^{\prime}\right\} \sim R^{5 / 4} \exp \left\{ \pm i c n^{1 / 2} \int^{t} R^{-5 / 2} d t^{\prime}\right\} .
$$

The distortion amplitude $a_{n}$ is then given by

$$
a_{n} \sim R^{-1 / 4} \exp \left\{ \pm i c n^{1 / 2} \int^{t} R^{-5 / 2} d t^{\prime}\right\}, \quad R \rightarrow 0 ;
$$

so that $a_{n}$ increases like $R^{-1 / 4}$ in amplitude and oscillates with increasing frequency as $R \rightarrow 0$. This behavior has been found by Birkhoff [5] by a different procedure. It is of interest that the instability found by Birkhoff near $R=0$ is qualitatively unaffected by surface tension.

The question remains over what range of $R$ is the linearized perturbation theory for the distortion amplitude*, $a$, valid or consistent. The following problem will therefore be solved. A spherical cavity with radius $R_{0}$ at $t=0$ expands, or collapses, from rest, $d R_{0} / d t=0$, under a constant pressure difference; at $t=0$, the cavity is supposed to have a distortion of small amplitude $a_{0}$, and the subsequent behavior of $a$ for any $R$ is to be determined. Complete solutions for this problem are readily found when surface tension is neglected and these solutions are given first. The effects of surface tension will then be illustrated by some special solutions.

(i) Expanding cavity; no surface tension

*The subscript $n$ for the distortion amplitude will be omitted in the following. 
With no surface tension, the stability equation to be solved simplifies to

$$
\frac{d^{2} a}{d t^{2}}+\frac{3}{R} \frac{d R}{d t} \frac{d a}{d t}-\frac{(n-1)}{R} \frac{d^{2} R}{d t^{2}} a=0 ;
$$

one finds from Eq. (6) that

$$
\left[\frac{d R}{d t}\right]^{2}=\frac{2 P}{3 \rho}\left[1-\frac{R_{0}^{3}}{R^{3}}\right]
$$

where $P=p_{i}-p_{0}>0$; and from Eq. (5) that

$$
\frac{d^{2} R}{d t^{2}}=\frac{P}{\rho} \frac{R_{0}^{3}}{R^{4}}
$$

If the independent variable in Eq. (17) is changed from $t$ to the volume ratio

$$
x=\frac{R_{0}^{3}}{R^{3}}, \quad 0 \leq x \leq 1 \text {; }
$$

there results

$$
x(1-x) \frac{d^{2} a}{d x^{2}}+\left[\frac{1}{3}-\frac{5}{6} x\right] \frac{d a}{d x}-\frac{(n-1)}{6} a=0 .
$$

Equation (21) will be recognized as the differential equation for the hypergeometric function $F(\alpha, \beta ; \gamma ; x)$ where the parameters have the values

$$
\begin{aligned}
& \alpha=\frac{-1+i(24 n-25)^{1 / 2}}{12}=-\frac{1}{12}+i \delta ; \\
& \beta=\frac{-1-i(24 n-25)^{1 / 2}}{12}=\alpha^{*} ; \\
& \gamma=\frac{1}{3} .
\end{aligned}
$$

It is convenient to take the general solution of Eq. (21) in the form [6]

$a=A F(\alpha, \beta ; 1 / 2 ; 1-x)+B(1-x)^{1 / 2} F(-\alpha+1 / 3,-\beta+1 / 3 ; 3 / 2 ; 1-x)$

where $A$ and $B$ are constants to be fixed by the initial conditions. If $a=a_{0}$ at $t=0$ or $R=R_{0}$, then one has

$$
A=a_{0}
$$

from Eq. (23). Similarly, if the initial velocity amplitude for the distortion is $v_{0}$, then

$$
\begin{aligned}
v_{0}=\left[\frac{d a}{d t}\right]_{t=0} & =\lim _{x \rightarrow 1}\left[\frac{d a}{d x} \frac{d x}{d t}\right], \\
& =\frac{3 B}{2 R_{0}}\left[\frac{2 P}{3 \rho}\right]^{1 / 2},
\end{aligned}
$$

which fixes the constant $B$. The quantity $(2 P / 3 \rho)^{1 / 2}$ is a characteristic velocity and $R_{0}$ is a characteristic length for the system, and it is convenient to describe the initial velocity amplitude in terms of the length

and Eq. (25) becomes

$$
l_{0}=\frac{v_{0} R_{0}}{(2 P / 3 \rho)^{1 / 2}}
$$

$$
B=2 l_{0} / 3 .
$$


The limiting value of $a$ as $R \rightarrow \infty$, or $x \rightarrow 0$, is readily determined from Eq. (23). One has

$$
a(R \rightarrow \infty)=a_{\infty}=\pi^{1 / 2} \Gamma(2 / 3)\left[\frac{a_{0}}{|\Gamma(1 / 2-\alpha)|^{2}}+\frac{l_{0}}{3|\Gamma(7 / 6+\alpha)|^{2}}\right]
$$

so that for large $n$

where from Eq. (22)

$$
a_{\infty} \sim \frac{1}{2} \pi^{-1 / 2} e^{\pi \delta} \Gamma(2 / 3)\left[a_{0} \delta^{-1 / 6}+\frac{l_{0}}{3} \delta^{-7 / 6}\right]
$$

$$
\delta=\frac{(24 n-25)^{1 / 2}}{12}
$$

Figure 1 shows the variation of $a / a_{0}$ with $R_{0} / R$ for various values of $n$ for the case in which the initial velocity amplitude is zero, $l_{0}=0$; Figure 2 shows the variation of $a / a_{0}$ for the case in which the initial velocity amplitude is different from zero. Of greater significance is the ratio of the distortion amplitude $a$ to the mean bubble radius $R$; the

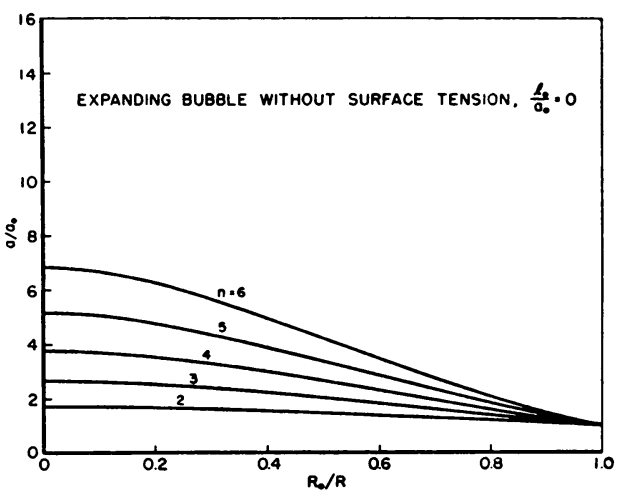

Fig. 1. The ratio of the distortion amplitude $a$ to its initial value $a_{0}$ is shown for an expanding vapor cavity as a function of $R_{0} / R$ where $R_{0}$ is the initial cavity radius and $R$ its radius at later times. The distortion of the spherical interface is $a_{n} Y_{n}$ where $Y_{n}$ is a spherical harmonic of order $n$. The initial velocity of the distortion is zero.

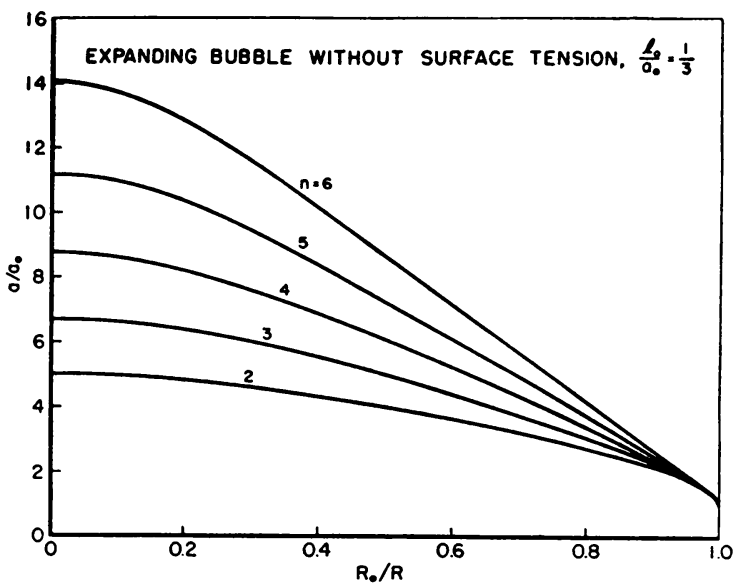

Fia. 2. The distortion amplitude is shown as a function of cavity radius for a non-zero initial distortion velocity. 
behavior of $\left(a / a_{0}\right)\left(R_{0} / R\right)$ is shown as a function of $R_{0} / R$ in Fig. 3 for the case in which the initial velocity amplitude is zero, and in Fig. 4 for the case in which the initial velocity amplitude is different from zero.

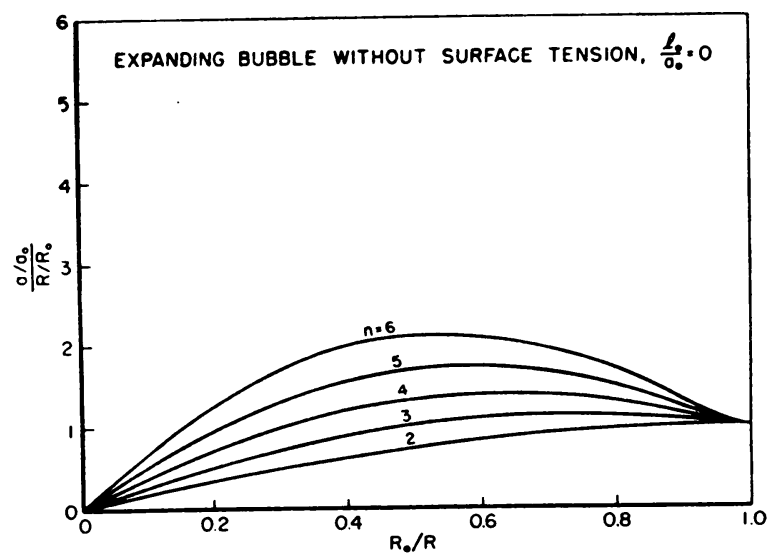

FIG. 3. The ratio of the distortion amplitude $a$ to the mean cavity radius $R$ (in units of $a_{0} / R_{0}$ ) is shown as a function of $R_{0} / R$ for the case shown in Fig. 1.

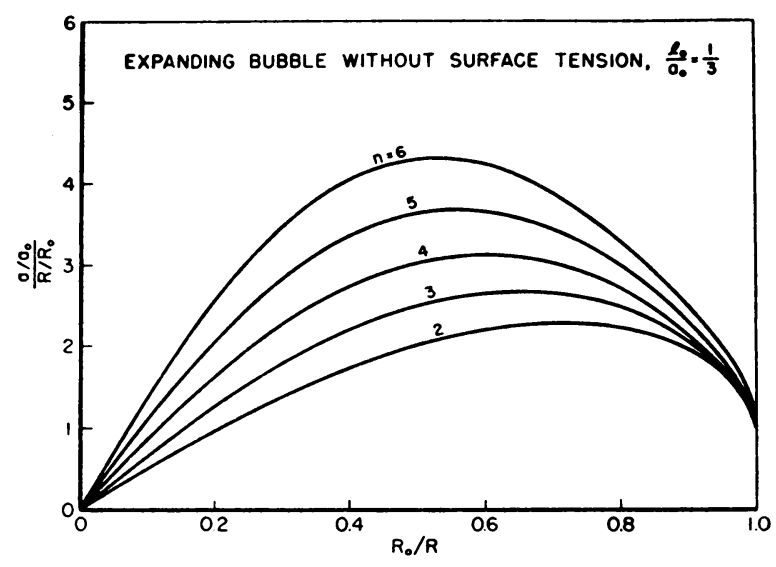

Fig. 4. The ratio of the distortion amplitude $a$ to the mean cavity radius $R$ (in units of $a_{0} / R_{0}$ ) is shown as a function of $R_{0} / R$ when the initial distortion amplitude velocity is non-zero.

(ii) Collapsing cavity; no surface tension

Equation (21) is also applicable to this case. It is convenient, however, to write the solution in the form [6]

$$
\begin{aligned}
a= & A x^{-\alpha} F(\alpha, \alpha+2 / 3 ; 1 / 2 ; 1-1 / x) \\
& +B x^{-\alpha}(1-1 / x)^{1 / 2} F(-\beta+1 / 3,-\beta+1 ; 3 / 2 ; 1-1 / x), \quad 1 \leq x \leq \infty .
\end{aligned}
$$

Equation (18) is written in the form

$$
\left[\frac{d R}{d t}\right]^{2}=\frac{2 p}{3 \rho}\left[\frac{R_{0}^{3}}{R^{3}}-1\right]
$$


where now $p=p_{0}-p_{i}>0$. The constants $A$ and $B$ are found as in the previous case in terms of the initial distortion amplitude $a_{0}$ and the initial velocity amplitude $v_{0}$ :

$$
\begin{aligned}
& A=a_{0} ; \\
& B=\frac{2}{3} v_{0} \frac{R_{0}}{(2 p / 3 \rho)^{1 / 2}}=\frac{2}{3} l_{0} .
\end{aligned}
$$

The solution may, of course, be written in a variety of forms. In place of Eq. (29) one may write, for example,

$a=A^{\prime} y^{\alpha} F(\alpha, \alpha+2 / 3 ; 2 \alpha+7 / 6 ; y)$

$$
+B^{\prime} y^{-\alpha-1 / 6} F(-\alpha+1 / 2,-\alpha-1 / 6 ;-2 \alpha+5 / 6 ; y),
$$

where now

$$
y=\frac{1}{x}=\frac{R^{3}}{R_{0}^{3}}, \quad 0 \leq y \leq 1
$$

$A^{\prime}$ and $B^{\prime}$ are linear combinations of $a_{0}$ and $l_{0}$ which will not be written explicitly here. From Eq. (30), one finds in the neighborhood of $y=0$ that

or

$$
a \approx A^{\prime} y^{-1 / 12+i \delta}+B^{\prime} y^{-1 / 12-i \delta},
$$

$$
a \approx \text { const } \times R^{-1 / 4} \text {, }
$$

which is the singularity noted by Birkhoff.

The variation of the distortion amplitude with mean bubble radius is shown in Fig. 5 for $n=3$ and in Fig. 6 for $n=6$. The quantity of significance is the ratio of $a$ to $R$; therefore, the variation of $\left(a / a_{0}\right)\left(R_{0} / R\right)$ with $R / R_{0}$ is shown in Fig. 7 for $n=3$ and in Fig. 8 for $n=6$.

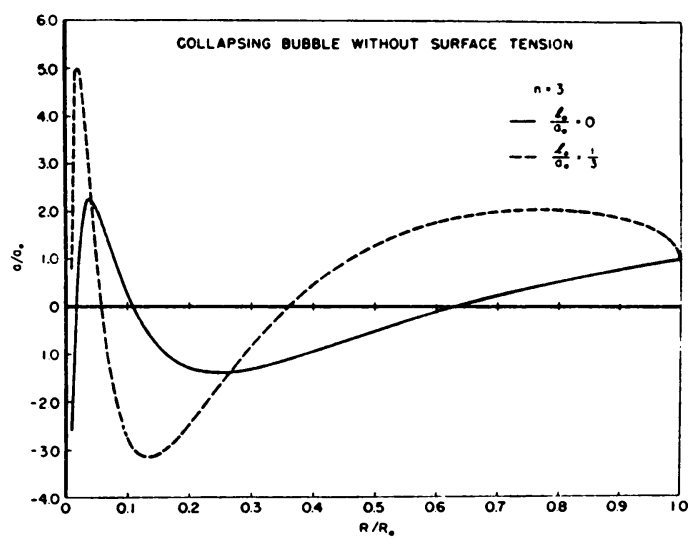

Fig. 5. The ratio of the distortion amplitude $a$ to its initial value $a_{0}$ is shown for a collapsing vapor cavity as a function of $R / R_{0}$. The case shown is for a distortion described by a spherical harmonic of order $n=3$. 


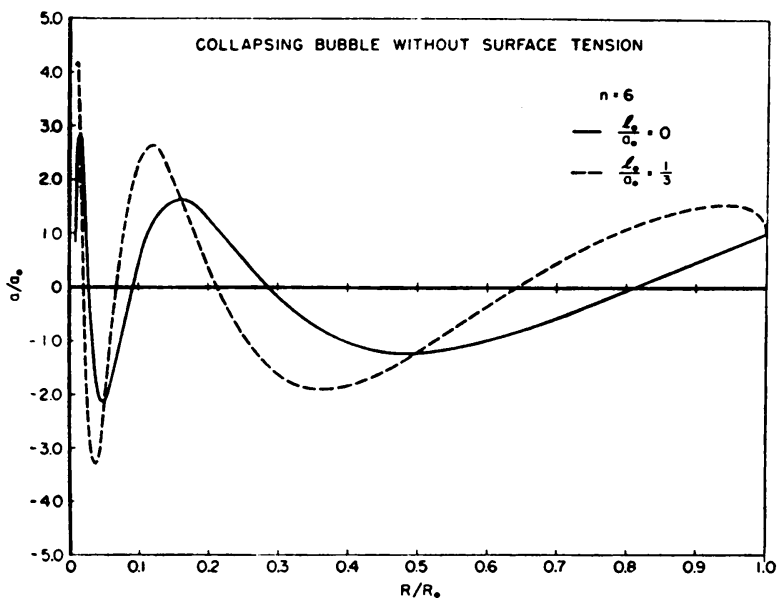

FIG. 6. The distortion amplitude is shown as a function of cavity radius for the case $n=6$.

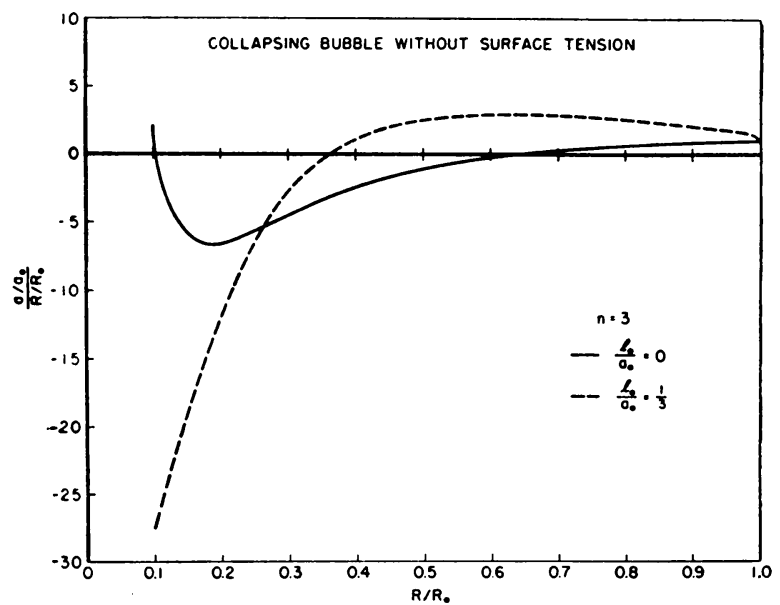

Frg. 7. The ratio of the distortion amplitude $a$ to the mean cavity radius $R$ (in units of $a_{0} / R_{0}$ ) is shown as a function of $R / R_{0}$ for $n=3$.

(iii) Expanding cavity with surface tension

For a bubble expanding from rest, $d R_{0} / d t=0$, one has from Eq. (6), if surface tension is included,

$$
\left[\frac{d R}{d t}\right]^{2}=\frac{2 P}{3 \rho}\left[1-\frac{R_{0}^{3}}{R^{3}}\right]-\frac{2 \sigma}{\rho R}\left[1-\frac{R_{0}^{2}}{R^{2}}\right]
$$

where $P=p_{i}-p_{0}>0$. The radial acceleration is determined by the relation

$$
R \frac{d^{2} R}{d t^{2}}+\frac{3}{2}\left[\frac{d R}{d t}\right]^{2}=\frac{P-2 \sigma / R}{\rho} .
$$

If the stability equation (2) is written in terms of the independent variable

$$
z=\frac{R_{0}}{R}
$$




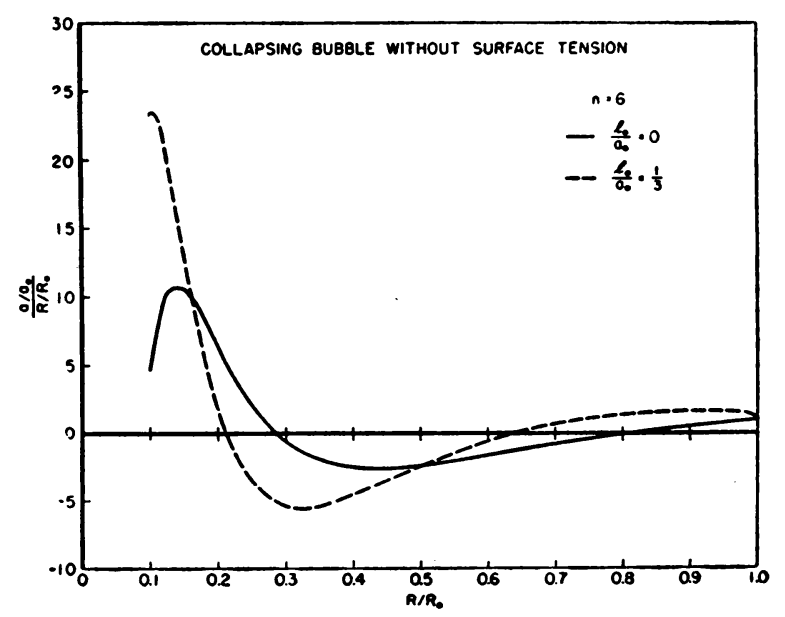

FIg. 8. The ratio of the distortion amplitude $a$ to the mean cavity radius $R$ (in units of $a_{0} / R_{0}$ ) is shown as a function of $R / R_{0}$ for $n=6$.

it becomes

$$
\begin{aligned}
\left\{\left\{\frac{2}{3}-k z+(k-2 / 3) z^{3}\right\}\right. & \frac{d^{2} a}{d z^{2}}-\left\{\frac{2}{3}-\frac{k}{2} z-\frac{1}{3}\left(\frac{3 k}{2}-1\right) z^{3}\right\} \frac{d a}{d z} \\
& -(n-1)\left\{\frac{k}{2}[1-(n+1)(n+2)]+\left(1-\frac{3 k}{2}\right) z^{2}\right\} a=0
\end{aligned}
$$

where

$$
k=\frac{2 \sigma}{R_{0} P}
$$

so that $k$ is the ratio of the initial value of the surface tension to the static pressure difference between the inside of the bubble and the liquid. Equation (35) has a neat solution for the special value of $k=2 / 3$ in which case it reduces to the hypergeometric differential equation. This value of $k$ is reasonable for vapor bubbles growing in superheated water where it is effectively slightly smaller than unity [7]. A convenient form for the solution is

$$
a=A F(\alpha, \beta ; 1 / 2 ; 1-z)+B(1-z)^{1 / 2} F(\alpha+1 / 2, \beta+1 / 2 ; 3 / 2 ; 1-z)
$$

where now

$$
\begin{aligned}
& \alpha=-\frac{3}{4}+\frac{(9+16 N)^{1 / 2}}{4} \\
& \beta=-\frac{3}{4}-\frac{(9+16 N)^{1 / 2}}{4}
\end{aligned}
$$

and

$$
N=\frac{(n-1)}{2}\left(n^{2}+3 n+1\right) \text {. }
$$

If $a_{0}$ is the initial distortion amplitude and $v_{0}$ the initial distortion velocity amplitude, then one finds

$$
A=a_{0},
$$


and

iwhere

$$
B=2 l_{0},
$$

$$
l_{0}=\frac{R_{0}}{(2 P / 3 \rho)^{1 / 2}} v_{0}
$$

The variation of $a / a_{0}$ with $R_{0} / R$ when $k=2 / 3$ is shown in Fig. 9 for $n=2$ and 3 . In Fig. 10, the variation of $\left(a / a_{0}\right)\left(R_{0} / R\right)$ with $R_{0} / R$ is shown for these same values of $n$.

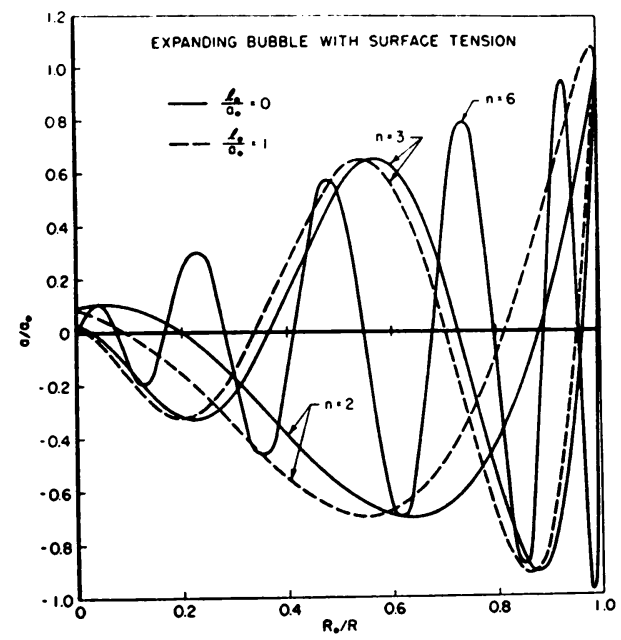

Frg. 9. The distortion amplitude $a$ relative to its initial value $a_{0}$ is shown for an expanding cavity as a function of $R_{0} / R$ for the case in which the effect of surface tension is included. For $n=6$ the curve with $l_{0} / a_{0}=1$ is not shown since it lies quite close to the curve $l_{0} / a_{0}=0$.

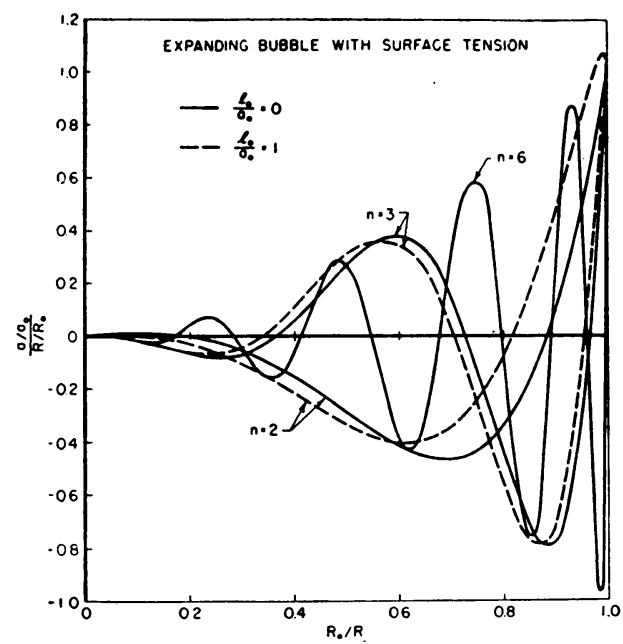

FIG. 10. The ratio of the distortion amplitude $a$ to the mean cavity radius (in units of $a_{0} / R_{0}$ ) is shown as a function of $R_{0} / R$ for the case in which the effect of surface tension is included. For $n=6$ the curves $l_{0} / a_{0}=1$ and $l_{0} / a_{0}=0$ are very near each other. 
When these curves are compared with Figs. 1 and 2, or Figs. 3 and 4, the stabilizing effect of surface tension is evident. It is also of interest to observe that, when surface tension is included, $a / a_{0}$ changes sign as $R$ increases.

(iv) Cavity collapse under surface tension alone

An additional case of special analytic simplicity occurs for $p_{i}=p_{0}$ so that the cavity collapses under the influence of surface tension alone. If the independent variable in the stability equation for $a$ is changed from $t$ to the area ratio

$$
u=\frac{R^{2}}{R_{0}^{2}}
$$

the solution of the resulting differential equation is readily found to be

$a=u^{m}\left[A F(\alpha, \beta ; 1 / 2 ; 1-u)+B(1-u)^{1 / 2} F(\alpha+1 / 2, \beta+1 / 2 ; 3 / 2 ; 1-u)\right]$,

where $m$ has the value

$$
m=\frac{-1+i(24 n-25)^{1 / 2}}{8}
$$

and $F$ is the hypergeometric function with parameters $\alpha$ and $\beta$ determined by the relations

$$
\begin{aligned}
\alpha \beta & =\frac{m}{2}-\frac{(n-1)}{8}\left(n^{2}+3 n+4\right) ; \\
\alpha+\beta & =2 m+\frac{3}{4} .
\end{aligned}
$$

If the initial distortion amplitude is $a_{0}$ and the initial distortion velocity amplitude is $v_{0}$, then

and

$$
A=a_{0}
$$

where

$$
B=-L_{0}
$$

$$
L_{0}=v_{0} \frac{R_{0}}{\left(2 \sigma / \rho R_{0}\right)^{1 / 2}}
$$

It is evident from Eq. (41) that

$$
a \rightarrow \text { const } \times R^{-1 / 4} \quad \text { as } \quad R \rightarrow 0 .
$$

3. Conclusion. For an expanding vapor cavity, an initially spherical shape is stable in the sense that the deformation amplitude $a$ remains small compared with $R$ if its initial value $a_{0}$ is small compared with the initial cavity radius $R_{0}$. The consistency and applicability of the linearized perturbation theory for the distortion amplitude is thus demonstrated. These conclusions from the linearized theory must be qualified for the case in which surface tension is negligible. As is shown graphically in Figs. 3 and 4, $a / R$ as a function of $R_{0} / R$ has a maximum which increases slowly with $n$, the order of the spherical harmonic. It follows, therefore, when surface tension is unimportant, that needlelike irregularities in the spherical interface may grow to significant amplitudes. The present linearized theory is inadequate to follow the development of these high 
order distortions of the interface. This instability for large $n$ disappears when surface tension is of significance so that no such restriction need be imposed on the applicability of the linearized theory in this case.

For a collapsing vapor cavity, on the other hand, the perturbation theory is valid provided the distortion amplitude is not followed to small cavity radii. If $R_{0}$ is the initial radius of the spherical cavity, then the distortion amplitudes remain small so long as $1 \geq R / R_{0} \geq 0.2$ where the lower limit is, of course, approximate. The linearized theory is thus applicable over an interesting and important range of cavity radius. As $R \rightarrow 0$, the distortion amplitudes oscillate in sign with increasing frequency and increase in magnitude like $R^{-1 / 4}$. This increase in distortion amplitude as $R^{-1 / 4}$ is found with and without surface tension. It may be remarked that the linearized perturbation theory for the distortion amplitudes breaks down in a range of radii near that for which the present model of the vapor cavity becomes invalid. It is known [8] that the vapor pressure within a collapsing vapor cavity, such as is encountered in cavitating flow, begins to rise very rapidly as $R / R_{0}$ becomes smaller than approximately 0.1 .

\section{BibLIOGRAPHY}

1. G. I. Taylor, Proc. Roy. Soc. (A)201, 192 (1950)

2. D. J. Lewis, Proc. Roy. Soc. (A)202, 81 (1950). See also R. E. Duff and H. T. Knight, Abstract S12, Bull. Am. Phys. Soc. 29, No. 1, Jan. 1954 (New York meeting)

3. M. S. Plesset, J. Appl. Phys. 25, 96 (1954)

4. W. G. Penney and A. T. Price, unpublished results, described briefly in R. H. Cole, Underwater explosions (Princeton University Press, 1948), p. 308

5. G. Birkhoff, in press. The authors are indebted to Professor Birkhoff for the opportunity of seeing his manuscript in advance of publication

6. Erdelyi, et al., Higher transcendental functions, vol. 1, pp. 105-6, McGraw-Hill, 1953

7. M. S. Plesset and S. A. Zwick, J. Appl. Phys. 25, 493 (1954)

8. S. A. Zwick and M. S. Plesset, J. of Math. and Phys. 33, 308 (1955) 